\title{
Unilateral Vestibular Schwannomas in Childhood without Evidence of Neurofibromatosis: Experience of 10 Patients at a Single Institute
}

\author{
Luxin YIN1', Zhenyu MA², Chunde $\mathrm{LI}^{2}$, Shiqi LUO² \\ ${ }^{1}$ The Affiliated Hospital of Xu Zhou Medical College, Department of Neurosurgery, Huaihai Xilu 99, Quanshan District, \\ Xuzhou, Jiangsu Province, China \\ ${ }^{2}$ Capital Medical University, Beijing Tiantan Hospital, Department of Neurosurgery, Beijing, China
}

\section{ABSTRACT}

AIM: We investigated the clinical presentation and management issues of unilateral vestibular schwannomas in childhood without evidence of neurofibromatosis.

MATERIAL and METHODS: Ten pediatric patients with unilateral vestibular schwannoma and no evidence of neurofibromatosis were treated by the senior author at Tiantan Hospital from January 1994 to December 2014. The clinical manifestations, neuroimaging findings, treatment methods, and therapeutic results were retrospectively reviewed.

RESULTS: The study included 6 male and 4 female patients. The mean age of the patients was 13.9 years (range, 11-15 years). Common clinical features included increased intracranial pressure, hearing loss, and ataxia. The median diameter of the tumors was $5.15 \mathrm{~cm}$. Gross total resection was achieved in 9 of 10 patients via a suboccipital retrosigmoid approach, and the perioperative mortality rate was $10 \%$. The follow-up period ranged from 1 to 12 years. One patient with facial nerve injury recovered to HouseBrackmann grade I postoperatively, 5 to grade II-III, and 2 to grade IV-V. Only 1 patient had preserved hearing.

CONCLUSION: The clinical features of pediatric vestibular schwannomas are different from those of adult vestibular schwannomas, and cranial nerve preservation in children is more difficult. When a child presents with hearing loss as well as cognitive disability, a vestibular schwannoma should be highly suspected. Hypervascular vestibular schwannomas in childhood should be managed by multistaged surgeries.

KEYWORDS: Vestibular schwannoma, Childhood, Diagnosis, Treatment

\section{INTRODUCTION}

$\mathrm{V}$ Testibular schwannomas, which originate from the vestibular division of cranial nerve VIII, occur more often in adults than children. Identification of a sporadic or non-neurofibromatosis type 2 vestibular schwannoma in a child is extremely rare (1-7). When a vestibular schwannoma is found in a child, neurofibromatosis should be the first differential diagnosis. We reviewed 10 cases involving $<16$-yearold patients without clinical evidence of neurofibromatosis (approximately $0.2 \%$ of all cases reported in the last 20 years in Beijing Tiantan Hospital).

\section{MATERIAL and METHODS}

\section{Patients}

Ten pediatric patients (6 male and 4 female patients) who were admitted from January 1994 to December 2014 and diagnosed with unilateral vestibular schwannomas were 
included in this study. The patients and their first-degree relatives were screened with gadolinium-enhanced brain and spinal cord magnetic resonance imaging (MRI), their skin was examined, and their eyes underwent slit-lamp examination for cataracts and Lisch nodules. None of the patients had a family history or any clinical evidence of neurofibromatosis.

Written informed consent for inclusion in the study was provided by each patient or the patient's legal representative. The study protocol was approved by the ethics committee of Beijing Tiantan Hospital.

\section{Neuroimaging Examination}

All patients were evaluated by preoperative computed tomography (CT), MRI, and regular postoperative follow-up $\mathrm{CT}$. Tumors were located in the left and right cerebellopontine angle area in 9 and 1 patient, respectively.

\section{Surgical Procedure}

In all cases, a suboccipital retrosigmoid approach was used with preoperative ventriculoperitoneal shunting in 1 patient and intraoperative ventricular puncture in 4 patients. For the other 5 patients, the cerebrospinal fluid was first released from the cisterna magna to reduce the intracranial pressure (ICP). The tumor was exposed and removed from the internal auditory canal. The patients' electrophysiological parameters were monitored during the operation. The patients' postoperative care after the surgery depended on the operative procedure. Patients were sent to the neurointensive care unit after the operation. Head CT in the immediate postoperative period after craniotomy was reserved for delayed recovery from anesthesia or unexpected acute neurologic deficits.

\section{Follow-up}

Patients were followed up by clinical examination and CT and/or MRI at 6 months and 1 year after surgery. Yearly clinical examination and MRI studies with gadolinium were subsequently carried out to evaluate the status of the contralateral cochleovestibular nerve and other components of the neural axis. Long-term follow-up data were collected from the outpatient files for most patients and by a telephone call or home visit for the other patients.

\section{RESULTS}

\section{Clinical Data}

Ten patients were diagnosed with unilateral vestibular schwannomas, including 6 boys and 4 girls (male:female ratio, 3:2) with a mean age of 13.9 years (range, 11-15 years). The mean duration of follow-up was 6.8 years (range, 1-12 years). The mean interval between the onset of symptoms and admission to our hospital was 1.4 years (range, 0.3-3 years). The clinical manifestations of unilateral vestibular schwannomas included signs of increased ICP in 9 patients, hearing loss in 6 patients, decreased hearing in 4 patients, tinnitus in 5 patients, and ataxia in 9 patients (Table I).

\section{Neuroimaging Findings}

In most patients, MRI revealed widened internal auditory canals, low signal intensity on T1-weighted imaging, and slightly higher signal intensity on T2-weighted imaging without cystic degeneration. Homogeneous enhancement was seen in all cases after contrast administration. The average diameter of the tumors was $5.15 \mathrm{~cm}$ (range, $3-8 \mathrm{~cm}$ ).

\section{Surgical Procedures}

Gross total surgical removal (GTR) (defined as $>95 \%$ resection by volume and occurring in 9 of 10 patients in our series) is the optimal therapy for symptomatic unilateral vestibular schwannomas in childhood (Figure 1A-D), although 1 patient died of a hematoma 3 days postoperatively because of a coagulation deficiency (Patient No. 2).

Subtotal removal (STR) (defined as $\leq 95 \%$ resection by volume and occurring in 1 of 10 patients in our series) was achieved in only 1 patient (Patient No. 1). During the first operation in Patient No. 1, we found that the tumor was firm, extremely vascular, and adherent to the brain stem in the cerebellopontine angle. Excessive bleeding made the dissection very difficult and slow. We decided to discontinue the procedure and complete the tumor removal during the second operation because the cerebellar hemisphere began to swell. After 2 years, the patient returned to our hospital for treatment of the residual tumor. On this occasion, we found that the tumor was avascular and separated much easier from the surrounding issues. GTR was achieved during the second operation, and no relapse was found until the latest follow-up; however, the facial and cochleovestibular nerves were not anatomically preserved.

\section{Follow-up}

Patients with GTR or STR were followed up for a median period of 6.8 years (range, 1-12 years). The facial nerve was anatomically preserved during the operation in all 8 patients with GTR. No tumor recurrence or clinical features of neurofibromatosis developed during the follow-up period (Figure 2A,B). The function of the facial nerve was recovered to House-Brackmann grade I in 1 patient, grade II-III in 5 patients, and grade IV-V in 2 patients. Hearing was preserved in only 1 of the 8 patients with GTR.

\section{DISCUSSION}

Unilateral vestibular schwannomas in children are a rarely seen pathological diagnosis (1-7), but the risk of neurofibromatosis type 2 must be considered. In this study, we reviewed 10 cases of unilateral vestibular schwannomas in children without clinical evidence of neurofibromatosis. The clinical features, imaging findings, treatment, and prognosis were discussed in this article.

The clinical features of vestibular schwannomas are more prominent in adults, who usually present with tinnitus, hearing loss, and balance disorder (8-10). Unlike in adults, although gradual hearing loss occurs in the early phase of the disease in children, it is usually mistaken for diseases such as otitis media. The duration of this delay in identification in children is usually months to years. In our series, the mean interval between the onset of symptoms and admission to our hospital was 1.4 
years (range, 0.3-3 years). Additionally, children frequently present with large tumors. In a meta-analysis of 1345 adult patients, the average size of the vestibular schwannomas was $1.18 \mathrm{~cm}$ (12), while the average diameter of the tumors in our series was $5.15 \mathrm{~cm}$ (range, 3-8 cm). A plausible explanation for this difference is that younger patients are unable to articulate the severity or characteristics of their symptoms in the same fashion as adults. The tumors are thus given attention only when they have grown sufficiently to generate hydrocephalus (hydrocephalus was found in 6 patients at the time of diagnosis and admission to our hospital).

In a report by Allcutt et al. (1), the initial symptoms of 3 patients were increased ICP with a mass effect on the brain stem, although 2 of these patients had lost their hearing. In a report by Pothula et al. (5), there were 2 cases of headaches with increased ICP and 1 case of balance disorder indicating damage to the cerebellum. In our study, the most common clinical features that initially precipitated a doctor's visit were increased ICP, facial paralysis, and symptoms related to the cerebellum or brain stem, although 6 patients had hearing loss and 4 had decreased hearing. As the disease progressed, increased ICP was observed in 9 patients and ataxia in 9 patients. Before surgery, facial paralysis was observed in 4 patients. The lower cranial nerves were involved in 4 patients, the trigeminal nerves in 4 patients, and limb weakness in 2 patients. These symptoms were caused by failure of early diagnosis. Continued growth of the tumor can lead to compression of the fourth ventricle and brain stem with attendant hydrocephalus, increased ICP, cerebellar and pyramidal syndromes, and lower cranial nerve palsies. Therefore, when a child presents with hearing loss and cognitive disability, a vestibular schwannoma should be highly suspected.

Vestibular schwannoma is benign, and because it is usually hypovascular, even a large tumor can be totally resected without major morbidity using recent microsurgical techniques (8-10). Although uncommon, rich abnormal tumor vessels can be associated with a hypervascular vestibular schwannoma, and the resulting excessive bleeding interferes with tumor resection. A hypervascular vestibular schwannoma is a unique clinical entity, and its management is difficult and different from that of the usual nonhypervascular vestibular schwannoma. Yamakami et al. (15) reviewed the clinical features and radiological findings of 78 unilateral vestibular schwannomas. They found that hypervascular vestibular schwannomas presented at a younger age than did non-hypervascular ones; i.e., hypervascular vestibular schwannomas were much more common in children. Allcutt et al. (1) reviewed 3 patients with unilateral vestibular schwannomas in childhood and found that all tumors were extremely vascular, unlike the majority of adult tumors. They encountered torrential hemorrhage during surgery in 2 of their patients, resulting in a prolonged operation, difficulty in identifying structures, and ultimately abandonment of the procedures when only partial

Table I: Patient Demographics and Clinical Features

\begin{tabular}{|c|c|c|c|c|c|c|c|c|}
\hline No. & Sex & $\begin{array}{c}\text { Age } \\
\text { (years) }\end{array}$ & $\begin{array}{l}\text { Initial } \\
\text { presentation }\end{array}$ & Symptoms & $\begin{array}{c}\text { Duration of } \\
\text { symptoms } \\
\text { (years) }\end{array}$ & $\begin{array}{l}\text { Side of tumor } \\
\text { (diameter) }\end{array}$ & Operation & $\begin{array}{l}\text { Surgical } \\
\text { approach }\end{array}$ \\
\hline 1 & $\mathrm{~F}$ & 15 & $\mathrm{HA}$ & $\begin{array}{c}\text { HA, PE, VA, V, VI, VIII, IX paralysis, } \\
\text { HL, CS, ataxia, FP }\end{array}$ & 3 & $\begin{array}{c}\mathrm{L}(7 \mathrm{~cm}) \\
\text { hydrocephalus }\end{array}$ & STR-GTR & $\mathrm{RS}$ \\
\hline 2 & M & 11 & $\mathrm{HA}$ & $\begin{array}{c}\mathrm{HA}, \mathrm{VA}, \mathrm{HL}, \mathrm{PE}, \mathrm{CS}, \mathrm{V}, \mathrm{VIII}, \mathrm{IX} \\
\text { paralysis, ataxia }\end{array}$ & 1 & $\begin{array}{l}\mathrm{L}(5 \mathrm{~cm}) \\
\text { hydrocephalus }\end{array}$ & GTR & $\mathrm{RS}$ \\
\hline 3 & M & 15 & $\mathrm{FP}$ & $\begin{array}{c}\text { HA, FP, HL, PE, V, VIII, IX paralysis, } \\
\text { CS, ataxia }\end{array}$ & 0.5 & $\begin{array}{c}\mathrm{L}(6 \mathrm{~cm}) \text {, } \\
\text { hydrocephalus }\end{array}$ & GTR & RS \\
\hline 4 & M & 15 & tinnitus & $\begin{array}{l}\text { Tinnitus, ache in the left side face, } \\
\text { DH }\end{array}$ & 2 & $\mathrm{~L}(3 \mathrm{~cm})$ & GTR & RS \\
\hline 5 & $\mathrm{~F}$ & 14 & tinnitus & $\begin{array}{l}\text { HA, tinnitus, double vision, } \mathrm{PE} \text {, } \\
\text { ataxia, HL, faint, V, VIII, IX paralysis }\end{array}$ & 0.3 & $\begin{array}{c}\mathrm{L}(6 \mathrm{~cm}) \text {, } \\
\text { hydrocephalus }\end{array}$ & GTR & $\mathrm{RS}$ \\
\hline 6 & $\mathrm{~F}$ & 15 & FP & FP, HA, PE, HL, ataxia & 0.5 & $\mathrm{~L}(5 \mathrm{~cm})$ & GTR & RS \\
\hline 7 & $\mathrm{M}$ & 15 & tinnitus & Tinnitus, HL, ataxia, PE, HA & 1 & $\begin{array}{l}\mathrm{L}(5 \mathrm{~cm}) \text {, } \\
\text { hydrocephalus }\end{array}$ & GTR & RS \\
\hline 8 & M & 12 & $\mathrm{FP}$ & $\begin{array}{c}\text { HA, ataxia, tinnitus, } \mathrm{PE} \text {, faint, } \\
\text { nystagmus, } \mathrm{DH}\end{array}$ & 2 & $\mathrm{~L}(4 \mathrm{~cm})$ & GTR & RS \\
\hline 9 & $\mathrm{~F}$ & 14 & $\mathrm{HA}$ & $\mathrm{HA}, \mathrm{DH}$, ataxia, $\mathrm{PE}$, nystagmus & 1 & $\begin{array}{c}\mathrm{L}(5 \mathrm{~cm}) \text {, } \\
\text { hydrocephalus }\end{array}$ & GTR & RS \\
\hline 10 & $\mathrm{M}$ & 13 & tinnitus & Tinnitus, DH, HA, ataxia, PE, FP & 3 & $\mathrm{R}(4.5 \mathrm{~cm})$ & GTR & RS \\
\hline
\end{tabular}

F: Female, M: Male, HA: Headache, DH: Decreased hearing, HL: Hearing loss, VA: Vomiting, PE: Papilledema, CS: Cerebellar symptom, L: Left, R: Right, GTR: Gross total resection, STR: Subtotal resection, RS: Retrosigmoid approach. 

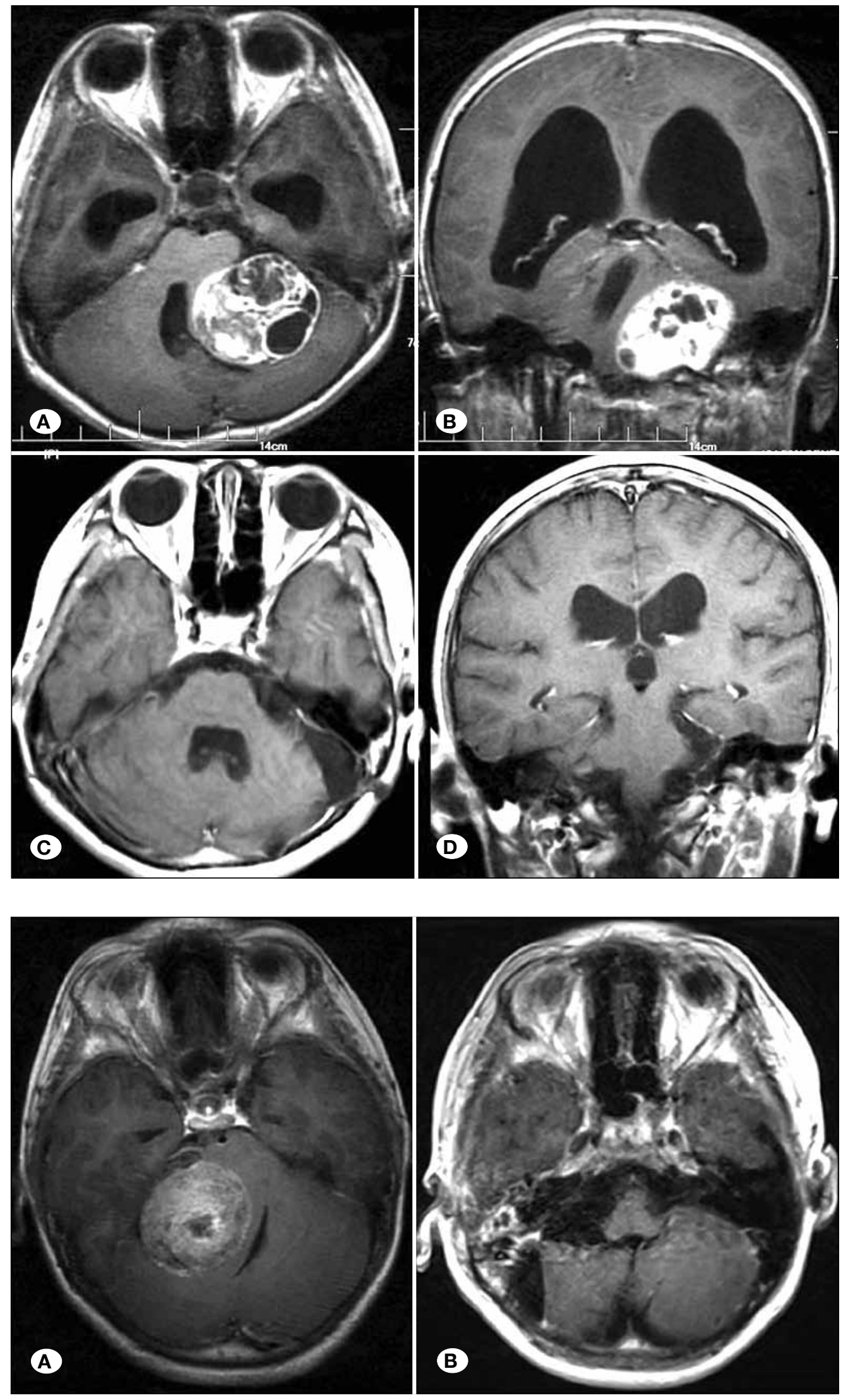

Figure 1: A, B) Preoperative MRI scan (axial and coronal views) with gadolinium showed a large acoustic neuroma in the left cerebellopontine angle area. C, D) Postoperative MRI scan (axial and coronal views) with gadolinium taken 2 years postoperatively showed that the tumor was completely absent without relapse.

Figure 2: A) Preoperative MRI scan (axial view) with gadolinium showed a large acoustic neuroma in the right cerebellopontine angle area. B) Postoperative MRI scan (axial view) with gadolinium taken 2 years postoperatively. 
resection had been completed. The authors recommended preoperative embolization for vestibular schwannomas with abundant feeding blood vessels in children. Of the 3 patients described by Pothula et al. (5), 1 had abundant feeding blood vessels with blood loss up to $2000 \mathrm{ml}$ during the operation. In contrast, Mazzoni et al. (4) reviewed 10 patients with vestibular schwannomas in childhood but did not encounter the problem of increased tumor vascularity and consequent intraoperative bleeding in any of the 10 cases. However, the average diameter of the tumors in their series was only 2.15 $\mathrm{cm}$, which was smaller than that in other series.

Abundant feeding blood vessels were present during the surgery in 1 of our patients. In Patient No. 1, the tumor measured $6 \times 5 \times 5 \mathrm{~cm}$ and had a pink surface and hard texture. Hemorrhage occurred during the first operation, making bipolar coagulation difficult and resulting in difficulty identifying structures. The procedure was ultimately abandoned when only $50 \%$ of the resection had been completed. After 2 years, the patient was readmitted for treatment of the residual tumor, which had a grayish color, a yellowish soft inner core, and was relatively hypovascular. The tumor was resected completely. The patient recovered well after the second operation and was discharged 18 days later.

Angiography of hypervascular acoustic tumors often shows extensive tumor vessels and tumor staining and early filling of large draining veins. However, the present study showed that the vascular supply of most hypervascular vestibular schwannomas were from the vertebrobasilar arteries. Preoperative embolization of these arteries carries a high risk and is not indicated for hypervascular acoustic tumors because reflux of particulate matter into the internal carotid circulation could result in infarction $(7,15)$. If difficult bleeding is encountered at the time of initial removal, as our Patient No. 1, stopping the operation and operating later should be considered. In our opinion, a hypervascular vestibular schwannoma should be managed by multistaged surgeries because after partial tumor removal in the first surgery, the remaining tumor might be less vascular, and both tumor bleeding and surgical risk are diminished in the second surgery $(3,5,7,15)$.

Vestibular schwannomas in children are usually full-grown with abundant feeding blood vessels at presentation; thus, there is a higher risk of facial nerve damage at surgery. Of 1000 cases of vestibular schwannomas reported by Samii and Matthies (8-10), the cochlear nerve was physically preserved in $68.0 \%$, the facial nerve was preserved in $93.0 \%$, and full tumor resection was performed in $97.9 \%$. In our series, the retrosigmoid approach was used in all 10 patients as reported by Mazzoni et al. (4). One patient with facial nerve injury recovered to House-Brackmann grade I postoperatively, 5 to grade II-III, and 2 to grade IV-V. Temporary paralysis also occurred after surgery in the patient with the largest tumor, which measured $60 \mathrm{~mm}$ in diameter. The patient gradually recovered full function. Mazzoni et al. (4) concluded that it is easier to preserve hearing in children than in adults. However, the tumor size was small in most of their patients, with an average diameter of $2.15 \mathrm{~cm}$. Additionally, there was no cranial nerve damage or cerebellar symptoms in their series.
In comparison, 2 of 3 patients described by Allcutt et al. (1) had damage to cranial nerves $\mathrm{V}, \mathrm{VI}$, and $\mathrm{VII}$, and 1patient sustained damage to cranial nerve IX.

In our study, patients with tumors measuring $3 \mathrm{~cm}$ in diameter had preservation of both the acoustic and facial nerves. The other 9 patients with tumors averaging $5.3 \mathrm{~cm}$ in diameter had hearing loss but facial nerve preservation. No relapse occurred in any of the surviving patients during the follow-up period.

Although recurrence of vestibular schwannomas after GTR is rare, microscopic fragments of the tumor may still be viable and propagate if given proper vascularization $(11,14)$. The role of stereotactic radiotherapy after microsurgery has been shown to be an effective measure in the treatment of both tumor regrowth and for use after STR, but the current treatment recommendations for children vary and are often limited by the significant neurotoxicity associated with radiation. In general, it is considered safe to observe the patient when postoperative CT or MRI shows GTR. Stereotactic radiotherapy has been recommended after the initial surgery in pediatric patients who have undergone STR $(6,11-14)$. Close observation with MRI should be performed in these patients. The success of this surgery-biased strategy depends on conscientious postoperative MRI follow-up and re-exploration if recurrence or progression is detected.

One possible complication to be avoided is cerebrospinal fluid leakage. This happened in $9.2 \%$ of the cases reported by Samii and Matthies (8-10). In another study by Mazzoni et al. (4), only 1 of 10 patients developed this complication. In contrast, no cerebrospinal fluid leakage occurred in the reports by Allcutt et al. (1), Pothula et al. (5), and the present study. The absence of this complication is probably associated with incomplete development of mastoid air cells in childhood. Another complication is hematoma. This occurred in $2.2 \%$ of the cases reported by Samii and Matthies (8-10). There was no such complication in the reports by Allcutt et al. (1) or Pothula et al. (5). There was only 1 case of hematoma in our study; it resulted in the patient's death and was probably secondary to a coagulation dysfunction.

We obtained no evidence of neurofibromatosis in any patients of the present study. However, we could not completely deny the possibility of a vestibular schwannoma developing on the contralateral side in the future. Follow-up MRI studies with gadolinium should include examination of the contralateral cranial nerve VIII to rule out the other cranial nerve tumors and meningiomas.

\section{- CONCLUSION}

The clinical features of pediatric vestibular schwannomas are different from those of adult vestibular schwannomas, and preservation of the cranial nerves is more difficult in children. Our study emphasizes the importance of early diagnosis and treatment of childhood vestibular schwannomas. When a child presents with hearing loss and cognitive disability, a vestibular schwannoma should be highly suspected. Hypervascular vestibular schwannoma in childhood should be managed by multi-staged surgeries. 


\section{REFERENCES}

1. Allcutt DA, Hoffman HJ, Isla A, Becker LE, Humphreys RP: Acoustic schwannomas in children. Neurosurgery 29:14-18, 1991.

2. Chen TC, Maceri DR, Giannotta SL, Shih L, McComb JG: Unilateral acoustic neuromas in childhood without evidence of neurofibromatosis: Case report and review of the literature. Am J Otol 13:318-322, 1992

3. Ishikawa K, Yasui N, Monoh K, Tada H, Mineura K, Sasajima $\mathrm{H}$, Togawa $\mathrm{K}$ : Unilateral acoustic neuroma in childhood. Auris Nasus Larynx 24: 99-104, 2007

4. Mazzoni A, Dubey SP, Poletti AM, Freeman S, Thorne J, Mawman D, O'Driscoll MP, Evans DG, Ramsden RT: Sporadic acoustic neuroma in pediatric patients. Int J Pediatr Otorhinolaryngol 71:1569-1572, 2007

5. Pothula VB, Lesser T, Mallucci C, May P, Foy P: Vestibular schwannomas in children. Otol Neurotol 22: 903-907, 2001

6. Roche PH, Robitail S, Delsanti C, Marouf R, Pellet W, Régis J: Radiosurgery of vestibular schwannomas after microsurgery and combined radio-microsurgery. Neurochirurgie 50:394400, 2004

7. Rushworth RG, Sorby WA, Smith SF: Acoustic neuroma in a child treated with the aid of preoperative arterial embolization. J Neurosurg 61: 396-398, 1984

8. Samii M, Matthies C: Management of 1000 vestibular schwannomas (acoustic neuromas): Hearing function in 1000 tumor resections. Neurosurgery 40:248-262,1997
9. Samii M, Matthies C: Management of 1000 vestibular schwannomas (acoustic neuromas): The facial nerve-preservation and restitution of function. Neurosurgery 40: 684-695, 1997

10. Samii M, Matthies C: Management of 1000 vestibular schwannomas (acoustic neuromas): Surgical management and results with an emphasis on complications and how to avoid them. Neurosurgery 40:11-23, 1997

11. Schmerber S, Palombi O, Bougagra K, Charchon R, Chirossel J, Gay E: Long-term control of vestibular schwannoma after a translabyrinthine complete removal. Neurosurgery 57:693698, 2005

12. Smouha EE, Yoo M, Mohr K, Davis RP: Conservative management of acoustic neuroma: A meta-analysis and proposed treatment algorithm. Laryngoscope 115: 450-454, 2005

13. Unger F, Walch C, Papaefthymiou G, Feichtinger K, Trummer M, Pendl G: Radiosurgery of residual and recurrent vestibular schwannomas. Acta Neurochir (Wien) 144: 671-676, 2002

14. Walcott BP, Sivarajan G, Bashinskaya B, Anderson DE, Leonetti JP, Origitano TC: Sporadic unilateral vestibular schwannoma in the pediatric population. J Neurosurg Pediatr 4:125-129, 2009

15. Yamakami I, Kobayashi E, Iwadate Y, Saeki N, Yamaura A: Hypervascular vestibular schwannomas. Surg Neurol 57:105112, 2002 